\title{
Health-related quality of life analysis in stage III melanoma patients treated with adjuvant dendritic cell therapy
}

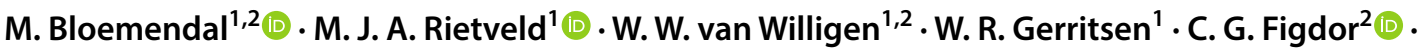

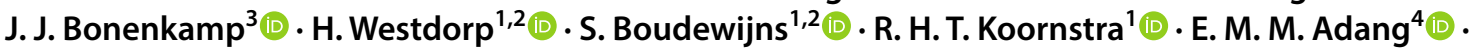

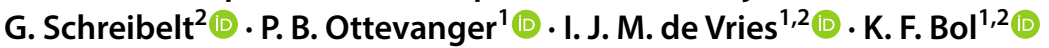

Received: 21 September 2018 / Accepted: 9 November 2018 / Published online: 21 November 2018

(c) The Author(s) 2018

\begin{abstract}
Background Health-related quality of life (HRQoL) is an important issue in the rapidly evolving field of adjuvant treatment for stage III melanoma. Dendritic cell vaccination is one of the adjuvant forms of therapy currently investigated.

Methods We enrolled adults with stage III melanoma to receive adjuvant dendritic cell vaccination after a complete radical lymph node dissection. HRQoL assessment was one of the secondary endpoints of this trial and investigated with the EORTC-QLQ-C30 questionnaire at baseline and week 26.

Results Fifteen patients with a median age of 50 years were included in the study, with twelve evaluable patients on study at time of the second questionnaire. Global health status and role functioning improved clinically relevant with a mean difference of $15(p=0.010)$ and 26 points $(p=0.005)$, respectively.

Discussion Despite the small number of patients, we found a clinically relevant improved global health status. Besides, compared to the other investigated therapies, toxicity of dendritic cell vaccination is low, which supports our finding.

Conclusion This is the first description of HRQoL in melanoma patients receiving dendritic cell vaccination. We show the expected improvement in global health status after surgical treatment of stage III melanoma. Thus, adjuvant dendritic cell vaccination does not seem to hamper this improvement, as shown in our small explorative study.
\end{abstract}

Keywords Dendritic cell vaccination $\cdot$ Immunotherapy $\cdot$ Adjuvant therapy $\cdot$ Melanoma $\cdot$ Stage III melanoma $\cdot$ Cancer

\section{Background}

The incidence of melanoma is increasing and in 2012 over 20,000 persons in Europe died due to melanoma [1,2]. Patients with regional lymph node metastasis, but without

M. J. A. Rietveld and W. W van Willigen have contributed equally to this work.

I. J. M. de Vries

jolanda.devries@radboudumc.nl

1 Department of Medical Oncology, Radboud University Medical Center, Nijmegen, The Netherlands

2 Department of Tumor Immunology, Radboud Institute for Molecular Life Sciences, Nijmegen, The Netherlands

3 Department of Surgery, Radboud University Medical Center, Nijmegen, The Netherlands

4 Department of Health Evidence, Radboud University Medical Center, Nijmegen, The Netherlands distant metastasis, are considered to have stage III disease. When operable, stage III melanoma is treated surgically and with curative intent. Until the recent publication of the MSLT-II trial, this included a radical lymph node dissection (RLND) in all patients [3]. Unfortunately, even after complete resection, stage III melanoma bears a high risk of recurrence with a 5-year overall survival (OS) rate of $78 \%, 59 \%$, and $40 \%$ in the substages IIIA, IIIB and IIIC, respectively [4]. Due to this high recurrence rate adjuvant treatment is warranted.

Current adjuvant treatment consists of radiotherapy to reduce the risk of local relapse in high-risk melanoma, without an effect on OS [5]. Clinical trials investigating adjuvant use of immune checkpoint inhibitors (ICI) and targeted therapies have been conducted. Both treatment with ipilimumab (anti-CTLA-4 antibody), and combined dabrafenib (BRAF inhibitor) and trametinib (MEK inhibitor) have shown a significant improved OS compared to placebo [6,7]. Nivolumab and pembrolizumab (both 
anti-PD-1 antibodies) were compared with ipilimumab and placebo, respectively. Both have shown a significant improved recurrence-free survival (RFS) compared to the control arm and data on OS are awaited [8,9]. At time of writing, ipilimumab, nivolumab, pembrolizumab as well as combined dabrafenib and trametinib are approved as adjuvant treatment by the Food and Drug Administration (FDA). The European Medicine Agency (EMA) approved use of adjuvant nivolumab and combined dabrafenib and trametinib. The Committee for Medicinal Products for Human Use (CHMP) gave a positive advise to the EMA about adjuvant use of pembrolizumab.

All these therapies have significant toxicity. Grade 3 or 4 adverse events occurred in $41.4 \%$ or $54.1 \%$ with dabrafenib/trametinib and ipilimumab, respectively [6, 7]. Nivolumab and pembrolizumab have less significant toxicity with $14.4 \%$ and $14.7 \%$ treatment-related grade 3 , 4 or 5 adverse events $[8,9]$. One patient receiving antiPD-1 treatment died, caused by pembrolizumab-induced myositis [9]. In contrast to the toxicity, assessment of the mean global health score in patients receiving adjuvant ipilimumab or nivolumab showed no clinically meaningful differences compared to baseline [8].

Dendritic cell (DC) vaccination is another form of immunotherapy, which seems to be more powerful in the adjuvant than metastatic setting, possibly due to the lower amount of tumor, hence less tumor-induced immune suppression [10]. This is supported by the improvement in OS we observed retrospectively in stage III melanoma patients vaccinated with DC, compared to matched controls receiving standard of care consisting of follow-up.

Adjuvant treatment options are emerging and survival benefits will be compared. Besides survival, it is important to take HRQoL into account since approximately half of stage III melanoma patients will not relapse without adjuvant treatment, but are exposed to the potential side effects [4]. In addition, a large number of patients are of working-age with a median age of 59 years (range 5-98) and therefore, normal life expectancy in this population is high [11]. In sharp contrast to ICI, severe toxicity is rare with DC vaccination and an advantage of this form of cellular immunotherapy, thus we hypothesized HRQoL is better with this form of therapy $[12,13]$.

To our knowledge, HRQoL in melanoma patients receiving DC-based therapy is not yet reported. Results from trials with DC vaccination in other forms of cancer do not show a negative impact on HRQoL. For example, in a trial with metastatic renal cell carcinoma patients, DC vaccination did not hamper HRQoL [14]. In another trial investigating patients with disseminated colorectal carcinoma receiving DC vaccination, HRQoL remained high and stable on most of the scales, except for general health perception and vitality [15]. Our study aims to investigate the HRQoL of stage III melanoma patients treated with adjuvant DC vaccination in our explorative study.

\section{Methods}

\section{Study design}

Prospective study, investigating HRQoL during adjuvant DC vaccination in patients with stage III melanoma, conducted at the Radboud University Nijmegen Medical Center. This study is registered as NCT02574377 at ClinicalTrials.gov. The protocol has been approved by the national Review Board (Central Committee on Research involving Human Subjects), and is in concordance with the Declaration of Helsinki and Good Clinical Practice. Written informed consent was obtained from all individual participants.

\section{Patients}

Eligibility criteria included histological documented stage III melanoma, complete RLND within 12 weeks before start of study and a WHO performance score of 0 or 1 . Patients with serious active infection, immunosuppressive conditions, or adjuvant radiotherapy were excluded. Immunological response was the primary endpoint of this study (manuscript in preparation). HRQoL analysis was one of the secondary endpoints.

\section{Methods}

Patients underwent an apheresis to acquire autologous mononuclear cells. Primary blood DC were collected from the apheresis product using the CliniMACS Prodigy and GMPgrade magnetic bead-coupled antibodies (Miltenyi Biotec, Bergisch Gladbach, Germany), matured with protaminemRNA, and loaded with peptides of gp100, tyrosinase, MAGE-A3, MAGE-C2 and NY-ESO1 [16]. After quality assessments, on average $3.6 \times 10^{6} \mathrm{DC}$ were injected under ultrasound guidance in a clinically tumor-free lymph node. One cycle consisted of 3 biweekly intranodal injections. In the absence of disease recurrence, two maintenance cycles of three injections each were given, with a 6-month interval between cycles as shown in Fig. 1. Toxicity assessment was performed before every vaccine, 1 week after a cycle as well as 3-5 weeks before and after the start of each cycle. Toxicity was scored according to the Common Terminology Criteria for Adverse Events, version 4.0.

\section{HRQoL assessment}

The European Organization for Research and Treatment of Cancer Quality of Life, Quality of Life core Questionnaire 
Fig. 1 Study schedule

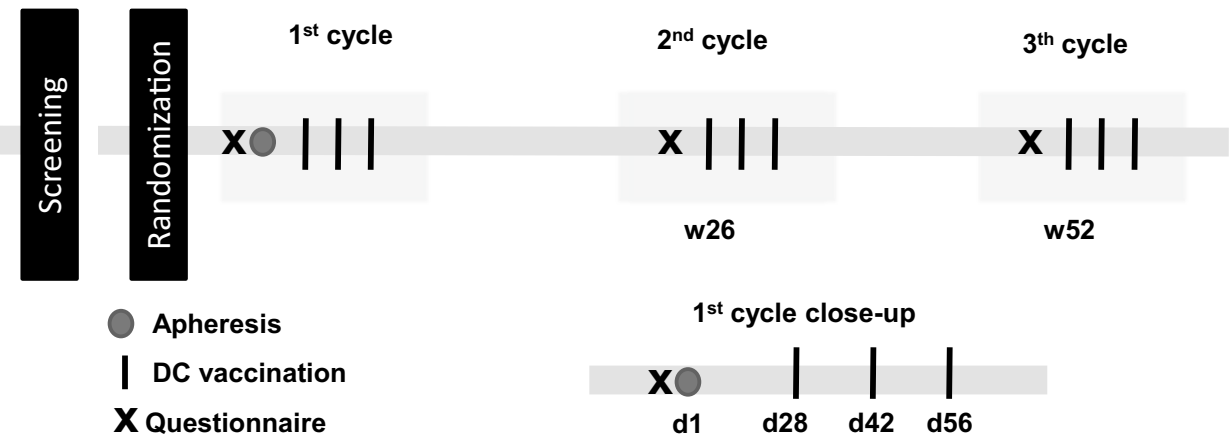

C30 (EORTC QLQ-C30) version 3.0 was used to assess HRQoL [17]. This 30-item questionnaire contains five functional scales on physical, role, emotional, cognitive, and social functioning, a global health status scale, three symptom scales on fatigue, nausea and vomiting, and pain, and six single items on dyspnea, insomnia, appetite loss, diarrhea, constipation and financial difficulties. The questions were framed as 'during the past week...' Response scales included: 'Not at all', 'A bit', 'Quite a bit', and 'Very much', except for the global health status scale, which ranges from 'Very poor' to 'Excellent'. Scores were linearly transformed to a 0-100 scale [18]. For the EORTC QLQ-C30, a higher score on the functioning-related domains represents a better quality of life and functioning; a higher score in one of the symptom-related domains represents a worse level of symptoms. Administration of the HRQoL questionnaires followed the clinical assessment schedule of the study, therefore, questionnaires were completed at baseline before apheresis, at the start of each subsequent cycle, and once a year thereafter for up to 5 years as shown in Fig. 1. Due to the small number of patients and high recurrence rate in this patient category, we report the HRQoL during the first cycle. The Cronbach's alpha-coefficient of our data is 0.86 , which suggests a good internal consistency.

\section{Statistical analysis}

To describe change in HRQoL during study participation, mean scale scores between patients at baseline and after 26 weeks, at the start of cycle 2, were compared using paired $t$ tests. All statistical analyses were conducted using SPSS Statistics version 22 (IBM Inc., Armonk, NY, USA). Figures were created with GraphPad Prism 5.03 (GraphPad Software, Inc., La Jolla, CA, USA). Besides reporting the statistical significant differences, considered as $p$ values of $<0.05$, more importantly we investigated clinically relevant differences. According to the work of Osoba et al. and King, a mean change of 10 points on a scale scores was defined as clinically relevant $[19,20]$. Missing items from multi-item scales of the EORTC QLQ-C30 were mean-imputed if at least half of the items from a scale were completed, according to the EORTC QLQ-C30 scoring guidelines [21].

\section{Results}

\section{Patient characteristics}

Fifteen patients were included in the study between October 2015 and August 2016. Two patients dropped out due to recurrent disease before week 26 and one patient was excluded from analysis due to completion of baseline questionnaire after apheresis. The remaining twelve patients who completed the questionnaire at baseline before apheresis and at week 26 were analyzed. Completion compliance was $100 \%$ for both time points and only one item was missing. Baseline characteristics are shown in Table 1. Median age of patients was 50 years (range 19-72 years). Seven patients were male (58\%) and five were female (42\%). All of

Table 1 Baseline characteristics $(n=12)$

\begin{tabular}{lc}
\hline Characteristic & \\
\hline Age [median-range (year)] & $50(19-72)$ \\
Sex $[n(\%)]$ & $7(58)$ \\
Male & $5(42)$ \\
Female & \\
WHO performance score $[n(\%)]$ & $2(17)$ \\
0 & $10(83)$ \\
1 & \\
Stage at inclusion $[n(\%)]$ & $3(25)$ \\
IIIA & $6(50)$ \\
IIIB & $2(17)$ \\
IIIC & $1(8)$ \\
IIIx & \\
Site of RLND $[n(\%)]$ & $1(8)$ \\
Head and neck & $3(25)$ \\
Axilla & $8(67)$ \\
Groin &
\end{tabular}

$R L N D$ radical lymph node dissection 
the AJCC 2009 stage III categories were represented. Most patients underwent an inguinal (67\%) or axillary (25\%) RLND and one patient underwent a neck RLND.

\section{Adverse events}

Adverse events, considered as possibly, probably or definitely related to study treatment, until week 26 are presented in Table 2. Patients may have had more than one adverse event. All adverse events were grade 1 or grade 2, except in one patient. The only grade 3 and 4 events were hypokalemia and hypocalcemia, respectively, both caused by apheresis. These disturbances were corrected with suppletion, and besides transient paresthesia, without clinical consequences.

\section{Functioning-related domains of HRQoL}

Except for cognitive functioning, all functioning-related domains showed an improvement over time, for role functioning this improvement was clinically relevant and significant. Mean scales scores of each of the scales of the EORTC QLQ-C30 questionnaire both at baseline and week 26 are presented in Fig. 2. Mean differences and clinical relevance are shown in Table 3. Physical and emotional functioning improved neither significant nor clinically relevant. Patients presented a clinically relevant and significant increase in role functioning 26 weeks after vaccination (mean difference 26 points, $p=0.005$ ). Social functioning showed a clinically

Table 2 Drug-related adverse events until week $26(n=12)$

\begin{tabular}{llll}
\hline Adverse event & $\begin{array}{l}\text { Any grade } \\
n(\%)\end{array}$ & $\begin{array}{l}\text { Grade } 3 \\
n(\%)\end{array}$ & $\begin{array}{l}\text { Grade } 4 \\
n(\%)\end{array}$ \\
\hline Any adverse event & $11(85)$ & $1(8)$ & $1(8)$ \\
Hypocalcemia & 0 & 0 & $1(8)$ \\
Hypokalemia & 0 & $1(8)$ & 0 \\
Fatigue & $8(67)$ & 0 & 0 \\
Flu like symptoms & $2(17)$ & 0 & 0 \\
Injection site reaction & $2(17)$ & 0 & 0 \\
Chills & $1(8)$ & 0 & 0 \\
Dizziness & $1(8)$ & 0 & 0 \\
Dry eye & $1(8)$ & 0 & 0 \\
Eosinophilia & $1(8)$ & 0 & 0 \\
GGT increased & $1(8)$ & 0 & 0 \\
Headache & $1(8)$ & 0 & 0 \\
Hypophosphatemia & $1(8)$ & 0 & 0 \\
Monocytosis & $1(8)$ & 0 & 0 \\
Paresthesia & $1(8)$ & 0 & 0 \\
Skin irritability & $1(8)$ & 0 & 0 \\
Urea elevated & $1(8)$ & 0 & 0 \\
\hline
\end{tabular}

Adverse events considered as possibly, probably or definitely related to the study drug relevant improvement with a mean difference of 16 points, but this difference was not significant $(p=0.082)$. Cognitive functioning was high at baseline with a mean score of 92 , and remained high at week 26 with a mean difference of 0 points $(p=1.000)$.

\section{Global health status}

The global health status scale showed a clinically relevant and significant increase of 15 points $(p=0.010)$. This indicates an improved global health status over time while receiving adjuvant DC-based therapy.

\section{Symptom-related domains of HRQoL}

Pain decreased significantly and clinically relevant with a mean difference of -19 points $(p=0.019)$. Besides, financial difficulties decreased significantly and clinically relevant as well, with a mean difference of -28 points $(p=0.017)$. None of the other symptom-related domains changed clinically relevant or significant.

\section{Follow-up}

At week 52 and week 78, 8 and 6 patients completed the questionnaire, respectively. None of the domains showed a clinically relevant and significant change during these follow-up visits.

\section{Discussion}

In our study, global health status of patients receiving adjuvant DC vaccination improved clinically relevant over time. In addition, role functioning, pain and financial difficulties showed a clinically relevant improvement. Although preliminary, this is promising data when compared to thus far available HRQoL data of ICI. After initial treatment, an increase in HRQoL was expected due to recovery from diagnosis and surgical treatment. In phase III trials investigating HRQoL of stage III melanoma patients while receiving adjuvant therapy, the observation or placebo arm indeed showed an increase in global health status over time, although not clinically relevant [5, 22-24]. We hypothesize that recovery from RLND is not hampered by the administration of DC vaccination.

\section{Clinical implications}

Other trials in the same study population do not report an improvement in global health status. The EORTC 18071 trial with adjuvant ipilimumab shows a decline of 4.2 points in global health status at week 24 compared to baseline [22]. 
Fig. 2 Mean scale scores $(0-100)$ with $95 \%$ confidence interval on functioning and symptom-related domains of each of the domains of the EORTC-QLQ-C30 at baseline and 26 weeks of treatment. *Clinically important and significant differences

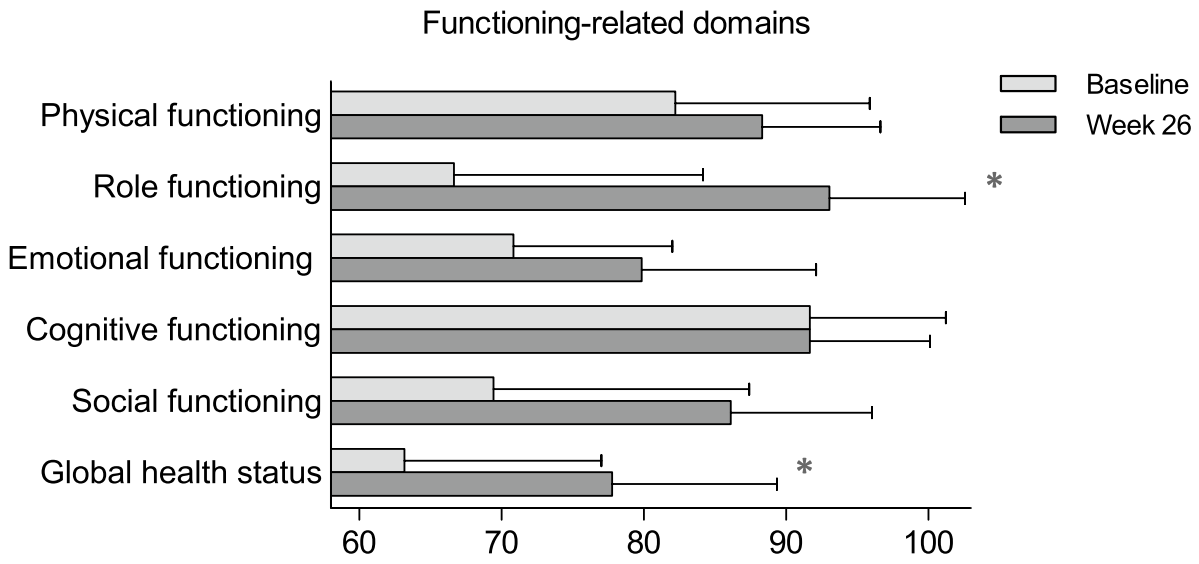

Symptom-related domains

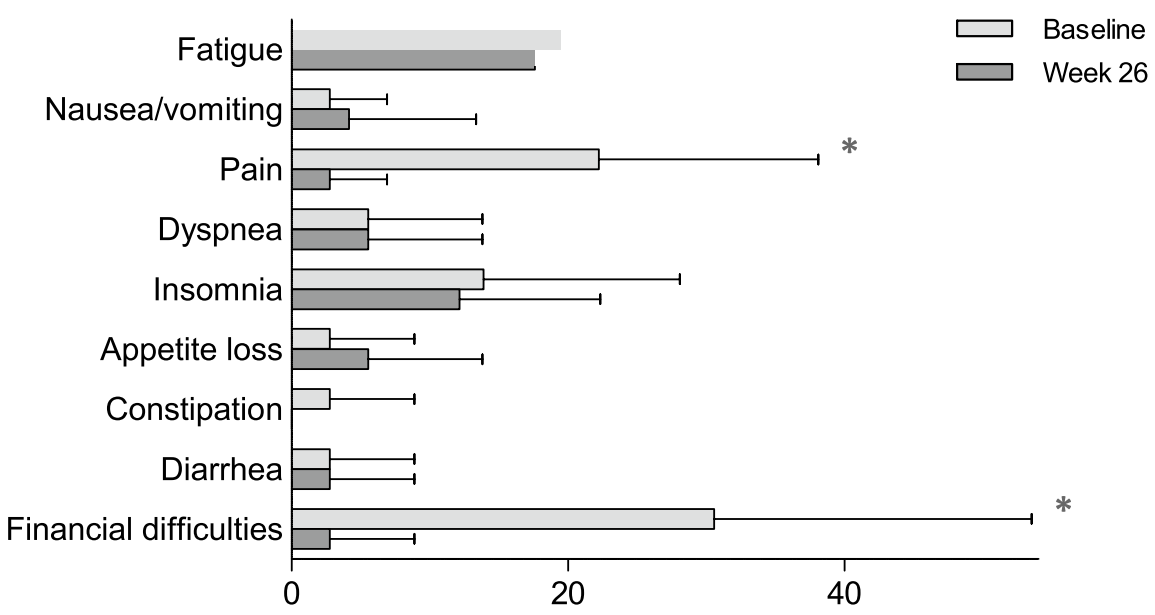

Lorigan and Green explain this contradiction between the large number of adverse events with ipilimumab and the relatively good HRQoL by the possibility of missed symptom-related HRQoL reduction caused by timing of the questionnaire. The HRQoL was assessed at week 24, and the last induction cycle was administered in the ninth week. Symptoms could have been missed since the median time to onset of adverse events is $4-12$ weeks and the median time to resolution is $4-8$ weeks. In addition, the possible perception of patients having adverse events as a positive reassurance they have been randomized to the treatment arm could have had an impact on the reported HRQoL [25]. The Checkmate-238 trial comparing adjuvant ipilimumab to nivolumab reports data of the global health status of included patients that does not differ clinically relevant from baseline [8]. At time of writing, quality of life data of the trial investigating combined dabrafenib and trametinib as well as the trial investigating pembrolizumab were not reported.

Besides global health status, financial difficulties improved clinically relevant and significant in our study. At baseline some patients reported difficulties with their financial situation due to their physical situation or medical treatment. In all but one of our patients, these difficulties have disappeared at week 26. We assume this is due to patients being able to work while receiving DC therapy, after recovery from RLND. Being able to work and participate in society is an important aspect of the quality of life for patients of working age with (cured) cancer [26]. The ability to work during treatment is also important for the cost-effectiveness of a treatment. Comparison of financial difficulties with other trials was not possible since the outcomes of this scale were not mentioned separately [8, 22-24, 27].

\section{Study limitations}

The major limitation of our study is the small number of patients. Nevertheless, we found a clinically relevant improvement of the global health status. Besides, compared to the other investigated therapies toxicity of DC vaccination is low, which supports our finding. Another limitation is the lack of a control arm. Participating in a therapeutic trial instead of a wait-and-see policy could have a positive impact 
Table 3 Mean scores [standard deviation (SD)] of HRQoL according to the different points in time

\begin{tabular}{|c|c|c|c|c|c|}
\hline & $\begin{array}{l}\text { Baseline } \\
\text { Score (SD) }\end{array}$ & $\begin{array}{l}\text { Week } 26 \\
\text { Score (SD) }\end{array}$ & $\begin{array}{l}\text { Mean difference } \\
\text { Points (SD) }\end{array}$ & $p$ value & CID \\
\hline \multicolumn{6}{|c|}{ QLQ-C30 functioning-related domains } \\
\hline Physical functioning & $82(22)$ & $88(13)$ & $6(14)$ & 0.152 & No \\
\hline Role functioning & $67(28)$ & $93(15)$ & $26(26)$ & 0.005 & Yes \\
\hline Emotional functioning & $71(18)$ & $80(19)$ & $9(19)$ & 0.127 & No \\
\hline Cognitive functioning & $92(15)$ & $92(13)$ & $0(17)$ & 1.000 & No \\
\hline Social functioning & $69(28)$ & $86(16)$ & $16(30)$ & 0.082 & Yes \\
\hline Global health status & $63(21)$ & $78(18)$ & $15(16)$ & 0.010 & Yes \\
\hline \multicolumn{6}{|c|}{ QLQ-C30 symptom-related domains } \\
\hline Fatigue & $19(24)$ & $18(20)$ & $-2(15)$ & 0.674 & No \\
\hline Nausea/vomiting & $3(6)$ & $4(14)$ & $1(11)$ & 0.674 & No \\
\hline Pain & $22(25)$ & $3(6)$ & $-19(24)$ & 0.019 & Yes \\
\hline Dyspnea & $6(13)$ & $6(13)$ & $0(14)$ & 1.000 & No \\
\hline Insomnia & $14(22)$ & $12(17)$ & $2(21)$ & 0.776 & No \\
\hline Appetite loss & $3(10)$ & $6(13)$ & $3(17)$ & 0.586 & No \\
\hline Constipation & $3(10)$ & $0(0)$ & $-3(10)$ & 0.339 & No \\
\hline Diarrhea & $3(10)$ & $3(10)$ & $0(14)$ & 1.000 & No \\
\hline Financial difficulties & $31(36)$ & $3(10)$ & $-28(34)$ & 0.017 & Yes \\
\hline
\end{tabular}

Mean HRQoL scores range from 0 to 100. A higher score on a functioning-related domain means better functioning, whereas a higher score on a symptom-related domain means more complaints

CID clinically important difference on the HRQoL. This bias could be avoided by inclusion of a placebo arm. Currently, we are investigating the HRQoL in our ongoing phase III trial, as further research is needed to confirm our findings.

\section{Conclusion}

This explorative study shows promising preliminary results with a clinically relevant improvement of the global health status. As further research on survival benefit, HRQoL and cost-effectiveness is required, we currently investigate these outcomes in our ongoing placebo-controlled phase III trial investigating adjuvant DC vaccination in stage IIIB and IIIC melanoma patients (NCT02993315). In this study, HRQoL is evaluated using the EORTC-QLQ-C30, the EQ-5D, and the Functional Assessment of Cancer Therapy-Melanoma questionnaires and cost-effectiveness using the Medical Consumption Questionnaire and the Short Health and Labour Questionnaire.

Acknowledgements The authors thank Annemiek J. de Boer, Kevin J.H. Bos, Tjitske Duiveman-de Boer, Ruud P.J. Hendrikx, Tom G.M. van Oorschot, Carlijn, J. Popelier, Jeanette M. Pots, Nicole M. Scharenborg, Mandy W.M.M. van de Rakt, and Valeska de Ruiter for analytic support, Simone M. Hins-de Bree for clinical support and the patients for completing the questionnaires.

Funding This work was supported by Dutch Cancer Society KWO Grant 2009-4402. C.G. Figdor received European Research Council
Advanced Grant PATHFINDER (269019) and the Netherlands Organization for Scientific Research Spinoza grant. I.J.M. de Vries received the Netherlands Organization for Scientific Research-Vici Grant (918.14.655).

\section{Compliance with ethical standards}

Conflict of interest The authors declare that they have no conflict of interest.

Ethical approval All procedures performed in studies involving human participants were in accordance with the ethical standards of the institutional and/or national research committee and with the 1964 Helsinki declaration and its later amendments or comparable ethical standards.

Informed consent Informed consent was obtained from all individual participants included in the study.

Open Access This article is distributed under the terms of the Creative Commons Attribution 4.0 International License (http://creativeco mmons.org/licenses/by/4.0/), which permits unrestricted use, distribution, and reproduction in any medium, provided you give appropriate credit to the original author(s) and the source, provide a link to the Creative Commons license, and indicate if changes were made.

\section{References}

1. Erdmann F, Lortet-Tieulent J, Schuz J, Zeeb H, Greinert R, Breitbart EW, et al. International trends in the incidence of malignant melanoma 1953-2008 - are recent generations at higher or lower 
risk? Int J Cancer. 2013;132(2):385-400. https://doi.org/10.1002/ ijc. 27616.

2. Estimated number of incident cases, both sexes, melanoma of skin, worldwide. Cancer Today International Agency for Research on Cancer. https://gco.iarc.fr (2012). Accessed 9 Jan 2018.

3. Faries MB, Thompson JF, Cochran AJ, Andtbacka RH, Mozzillo N, Zager JS, et al. Completion dissection or observation for sentinel-node metastasis in melanoma. N Engl J Med. 2017;376(23):2211-22. https://doi.org/10.1056/NEJMoa1613210.

4. Balch CM, Gershenwald JE, Soong S-J, Thompson JF, Atkins MB, Byrd DR, et al. Final version of 2009 AJCC melanoma staging and classification. J Clin Oncol. 2009;27(36):6199-206.

5. Henderson MA, Burmeister BH, Ainslie J, Fisher R, Di Iulio J, Smithers BM, et al. Adjuvant lymph-node field radiotherapy versus observation only in patients with melanoma at high risk of further lymph-node field relapse after lymphadenectomy (ANZMTG 01.02/TROG 02.01): 6-year follow-up of a phase 3, randomised controlled trial. Lancet Oncol. 2015;16(9):1049-60. https://doi. org/10.1016/S1470-2045(15)00187-4.

6. Eggermont AM, Chiarion-Sileni V, Grob JJ, Dummer R, Wolchok JD, Schmidt H, et al. Prolonged survival in stage III melanoma with ipilimumab adjuvant therapy. N Engl J Med. 2016. https:// doi.org/10.1056/nejmoa1611299.

7. Long GV, Hauschild A, Santinami M, Atkinson V, Mandala M, Chiarion-Sileni V, et al. Adjuvant dabrafenib plus trametinib in stage III BRAF-mutated melanoma. N Engl J Med. 2017. https:// doi.org/10.1056/nejmoa1708539.

8. Weber J, Mandala M, Del Vecchio M, Gogas HJ, Arance AM, Cowey CL, et al. Adjuvant nivolumab versus ipilimumab in resected stage III or IV melanoma. N Engl J Med. 2017. https:// doi.org/10.1056/nejmoa1709030.

9. Eggermont AMM, Blank CU, Mandala M, Long GV, Atkinson V, Dalle S, et al. Adjuvant pembrolizumab versus placebo in resected stage III melanoma. N Engl J Med. 2018. https://doi.org/10.1056/ nejmoa1802357.

10. Gajewski TF. Failure at the effector phase: immune barriers at the level of the melanoma tumor microenvironment. Clin Cancer Res. 2007;13(18 Pt 1):5256-61. https://doi.org/10.1158/1078-0432. CCR-07-0892.

11. Haydu LE, Scolyer RA, Lo S, Quinn MJ, Saw RPM, Shannon $\mathrm{KF}$, et al. Conditional survival: an assessment of the prognosis of patients at time points after initial diagnosis and treatment of locoregional melanoma metastasis. J Clin Oncol. 2017;35(15):1721-9. https://doi.org/10.1200/JCO.2016.71.9393.

12. Anguille S, Smits EL, Lion E, van Tendeloo VF, Berneman ZN. Clinical use of dendritic cells for cancer therapy. Lancet Oncol. 2014;15(7):e257-67. https://doi.org/10.1016/S1470 -2045(13)70585-0.

13. Draube A, Klein-Gonzalez N, Mattheus S, Brillant C, Hellmich $\mathrm{M}$, Engert A, et al. Dendritic cell based tumor vaccination in prostate and renal cell cancer: a systematic review and meta-analysis. PLoS ONE. 2011;6(4):e18801. https://doi.org/10.1371/journ al.pone.0018801.

14. Leonhartsberger N, Ramoner R, Falkensammer C, Rahm A, Gander H, Holtl L, et al. Quality of life during dendritic cell vaccination against metastatic renal cell carcinoma. Cancer Immunol Immunother. 2012;61(9):1407-13. https://doi.org/10.1007/s0026 2-012-1207-7.
15. Burgdorf SK, Fischer A, Myschetzky PS, Munksgaard SB, Zocca MB, Claesson MH, et al. Clinical responses in patients with advanced colorectal cancer to a dendritic cell based vaccine. Oncol Rep. 2008;20(6):1305-11.

16. Skold AE, van Beek JJ, Sittig SP, Bakdash G, Tel J, Schreibelt $\mathrm{G}$, et al. Protamine-stabilized RNA as an ex vivo stimulant of primary human dendritic cell subsets. Cancer Immunol Immunother. 2015;64(11):1461-73. https://doi.org/10.1007/s0026 2-015-1746-9.

17. Niezgoda HE, Pater JL. A validation-study of the domains of the core eortc quality-of-life questionnaire. Qual Life Res. 1993;2(5):319-25. https://doi.org/10.1007/Bf00449426.

18. Fayers PM. Interpreting quality of life data: populationbased reference data for the EORTC QLQ-C30. Eur J Cancer. 2001;37(11):1331-4. https://doi.org/10.1016/S0959 $-8049(01) 00127-7$.

19. Osoba D, Rodrigues G, Myles J, Zee B, Pater J. Interpreting the significance of changes in health-related quality-of-life scores. J Clin Oncol. 1998;16(1):139-44.

20. King MT. The interpretation of scores from the EORTC quality of life questionnaire QLQ-C30. Qual Life Res. 1996;5(6):555-67. https://doi.org/10.1007/Bf00439229.

21. Fayers PM, Aaronson NK, Bjordal K, Groenvold M, Curran D, Bottomley A. EORTC QLQ-C30 scoring manual. 3rd ed. Brussels: The EORTC Quality of Life Group; 2001.

22. Coens C, Suciu S, Chiarion-Sileni V, Grob JJ, Dummer R, Wolchok JD, et al. Health-related quality of life with adjuvant ipilimumab versus placebo after complete resection of high-risk stage III melanoma (EORTC 18071): secondary outcomes of a multinational, randomised, double-blind, phase 3 trial. Lancet Oncol. 2017;18(3):393-403. https://doi.org/10.1016/S1470 -2045(17)30015-3.

23. Bottomley A, Coens C, Suciu S, Santinami M, Kruit W, Testori A, et al. Adjuvant therapy with pegylated interferon alfa-2b versus observation in resected stage III melanoma: a phase III randomized controlled trial of health-related quality of life and symptoms by the European Organisation for Research and Treatment of Cancer Melanoma Group. J Clin Oncol. 2009;27(18):2916-23. https://doi.org/10.1200/JCO.2008.20.2069.

24. Garbe C, Radny P, Linse R, Dummer R, Gutzmer R, Ulrich J, et al. Adjuvant low-dose interferon \{alpha\}2a with or without dacarbazine compared with surgery alone: a prospective-randomized phase III DeCOG trial in melanoma patients with regional lymph node metastasis. Ann Oncol. 2008;19(6):1195-201. https://doi. org/10.1093/annonc/mdn001.

25. Lorigan P, Green AC. Immunotherapy: does adjuvant ipilimumab have little adverse effect on quality of life? Nat Rev Clin Oncol. 2017;14(7):395-6. https://doi.org/10.1038/nrclinonc.2017.60.

26. Peteet JR. Cancer and the meaning of work. Gen Hosp Psychiatry. 2000;22(3):200-5.

27. Mohr P, Hauschild A, Trefzer U, Enk A, Tilgen W, Loquai C, et al. Intermittent high-dose intravenous interferon alfa- $2 b$ for adjuvant treatment of stage III melanoma: final analysis of a randomized phase III dermatologic cooperative oncology group trial. J Clin Oncol. 2015;33(34):4077-84. https://doi.org/10.1200/ JCO.2014.59.6932. 